\title{
Asymmetric syntheses of (+)- and (-)-collybolide enable reevaluation of kappa-opioid receptor agonism
}

\author{
Sophia L. Shevick, ${ }^{1,3}$ Stephan M. Freeman, ${ }^{1,3}$ Guanghu Tong, ${ }^{\dagger}$ Robin J. Russo, ${ }^{2,3}$ Laura M. Bohn ${ }^{2}$ and \\ Ryan A. Shenvi ${ }^{1 *}$ \\ ${ }^{1}$ Department of Chemistry, Scripps Research, 10550 North Torrey Pines Road, La Jolla, California 92037, United States. \\ ${ }^{2}$ Department of Molecular Medicine, Scripps Research, 130 Scripps Way, Jupiter, FL 33458 \\ ${ }^{3}$ Skaggs Graduate School of Chemical and Biological Sciences
}

\begin{abstract}
The fungal metabolite collybolide attracted attention as a non-nitrogenous, potent and biased agonist of the kappa-opioid receptor (KOR). Here we report a 10-step asymmetric synthesis of this complex sesquiterpene that enables facile access to either enantiomer. The synthesis relies on a diastereoselective $\alpha$-benzoyloxylation to install the buried C6 benzoate and avoid irreversible translactonization of the congested, functionally dense core. Neither enantiomer, however, exhibited KOR agonism, indicating that collybolide has been mischaracterized as a KOR agonist and leaving open the basis for antipruritic effects in mice.
\end{abstract}

Introduction The freckled mushroom Collybia maculata (nom. alt. Rhodocollybia maculata) clusters in lignan-rich soils of conifer forests of Europe and North America. Bitter and tough to the point of inedibility, no references mention its use in traditional medicine nor its activity in mammals. In 2016, however, a metabolite of $C$. maculata ${ }^{1}$ was reported to exhibit potent and biased agonism of the kappa-opioid receptor (KOR). ${ }^{2}$ This sesquiterpene, collybolide, had been singled out for testing due to the similarity of its furano- $\delta$-lactone motif to that of the potent KOR agonist diterpene salvinorin A (SalA). In side-by-side assays with SalA, collybolide exhibited typical agonist behavior selective for the KOR receptor. In the GTP $\gamma \mathrm{S}$ binding assay, the collybolide-induced response was submaximal compared to SalA; while in the ERK1/2 map kinase and Akt phosphorylation studies, collybolide produced more stimulation than SalA. This change in relative rank-order efficacy led the authors to conclude that collybolide displays biased agonism at KOR. ${ }^{3}$ Collybolide also exhibited antipruritic activity in mice, a known consequence of KOR agonism. $^{4}$

The translational potential of collybolide attracted attention across the biomedical community ${ }^{5}$ due to indications for the KOR as a target in next-generation analgesics, antipruritics and antidepressants. ${ }^{6}$ However, many questions remained: the unusual dose-response curves and the lack of a $\beta$-arrestin recruitment assay, specifically, required greater pharmacological scrutiny, ${ }^{7}$ and structural homology with SalA was non-obvious in three dimensions (Figure 1). Synthetic access to collybolide might address these questions, clarify the absolute stereochemistry of $1,{ }^{8}$ provide material with a different impurity profile than $C$. maculata isolates (vide infra) and establish means for deep-seated modifications of the scaffold. Here, we report the first synthesis of collybolide and the biochemical profiles of both enantiomers. We find that neither (+)-1 nor (-)-1 agonizes KOR, raising the possibility that an unidentified contaminant of the $C$. maculata extract may agonize opioid receptors.
Degradation studies ${ }^{1 \mathrm{a}}$ of collybolide revealed the synthetic challenges that lay ahead. Like SalA, collybolide (1) epimerizes under acidic conditions, scrambling C7 stereochemistry. Treatment of $\mathbf{1}$ with alkali causes benzoate solvolysis and lactone cleavage. The nucleophilic oxygen (O5) of $\mathbf{1}$ is positioned $3.1 \AA$ from $\mathrm{C} 15$ and the isomer, neocollybolide (3), is calculated (MM2) to be $1.2 \mathrm{kcal} / \mathrm{mol}$ more stable than $\mathbf{1}$, resulting in kinetic and thermodynamic preference for the formation of $\mathbf{2}$ and $3{ }^{9}$

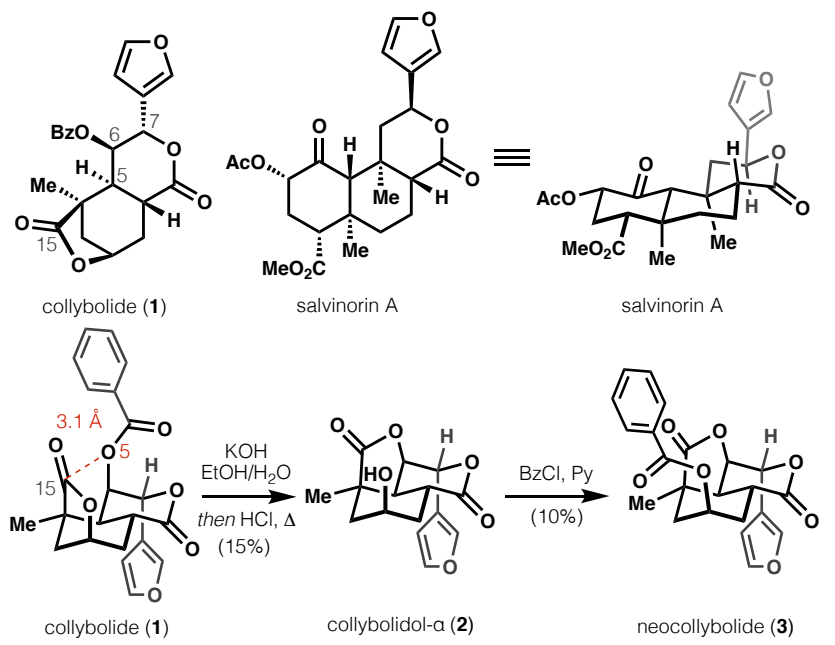

Figure 1. Collybolide and salvinorin A are suggested to share the $\kappa$-opioid receptor as a target, despite prominent differences in structure.

Treatment of $\mathbf{2}$ with benzoyl chloride delivers neocollybolide (3); rearrangement to the collybolide scaffold does not occur. The preference for $\mathbf{3}$ over $\mathbf{1}$ stands in contrast to reactivity of other sesquiterpenes like bilobalide where benzoylation of a hindered alcohol promotes scaffold rearrangement. ${ }^{10}$ Synthesis of the collybolide scaffold, therefore, cannot rely on rearrangement in favor of the desired scaffold; the preference for $\mathbf{2}$ would prove detrimental to our first-, second- 
and third-generation routes and dictate our ultimately successful approach to synthesize $\mathbf{1}$.

Our first retrosynthesis of collybolide identified $\mathrm{C} 6 / \mathrm{C} 7$ erythro-diol 5 as a sensible intermediate, accessible through epoxidation or dihydroxylation (and inversion) from vinyl furan 4 (Figure 2). ${ }^{11}$ Subjection of 4 to various oxidants including $\mathrm{OsO}_{4}$, however, resulted in furan oxidation. A secondgeneration approach accessed $\mathbf{5}$ via internal oxidation (see SI) but uncovered the tendency of the $\mathrm{C} 6$ alcohol (O5) to lactonize with $\mathrm{C} 15$, mirroring observations in the isolation literature that collybolidol- $\alpha$ (2) predominated upon saponification/acidification of $\mathbf{1}$. Similarly, a third route probed the dihydroxylation of intermediate 7 prior to furan installation and identified a rapid lactonization to form the thermodynamically-favored 5,6-fused $\gamma$-lactone 8 .

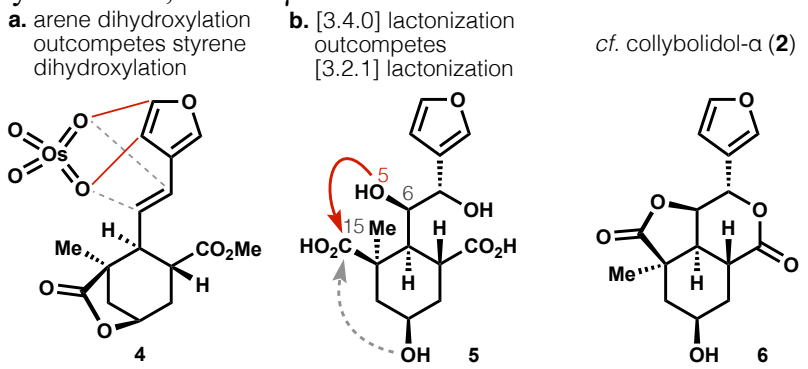

c. alkene dihydroxylation precedes fast lactonization d. C-OBz bond formation

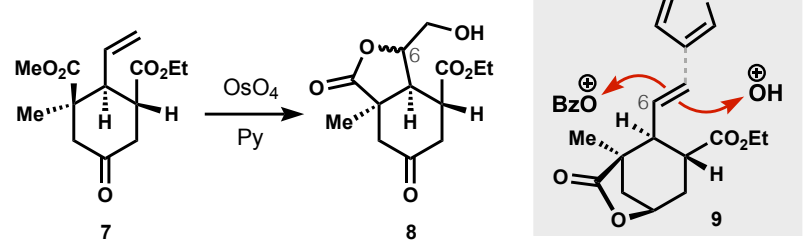

Figure 2. Three generations of routes reveal the difficulty of $\mathrm{C6}-\mathrm{OH}$ intermediates.

These three unsuccessful strategies called for an alternative approach that avoided the $\mathrm{C} 6$ alcohol entirely and resisted the reflexive benzoylation transform $\left(+\mathrm{Bz}^{+}\right)$suggested by 1 . This fourth-generation approach (Figure $2 \mathrm{~d}$ ) would install the C6 benzoyloxy group directly $\left(+\mathrm{BzO}^{+}\right)$, averting undesired translactonization and, ultimately, enabling the first chemical synthesis of $\mathbf{1}$. All four generations proceeded through the same densely functionalized, enantioenriched core 9. Like our third-generation route, furan installation would occur last, subsequent to alkene oxidation, to avoid furan decomposition. Benzoyloxylation, however, would require an electrophilic source of benzoate and its stereoselective addition to one alkene face - a high risk tactic that ultimately paid off.

Results and Discussion Synthesis commenced with an asymmetric, organocatalyzed cycloaddition, utilizing the Hayashi-Jørgensen diarylprolinol triethylsilyl ether ammonium catalyst $(R)-(-)-\mathbf{1 0}$, a more soluble variant of the commercially available trimethylsilyl ether. ${ }^{12,13}$ The electron-deficient diene 12 necessitated extended incubation with 11 (4 days at 4 $\left.{ }^{\circ} \mathrm{C}\right)$ but yielded cycloadduct $(-)-\mathbf{1 3}(80 \%, 92 \%$ ee $)$ in large quantity (12 g) after careful optimization. In contrast to previous work in which the ammonium salt was formed in situ, we pre-formed the air-stable triflate salt (-)-10, as small excesses of triflic acid promoted Mukaiyama-aldol side reactions. The exo-adduct (-)-13 was assigned definitively based on the homodecoupled ${ }^{1} \mathrm{H}$ NMR (homodec) coupling constant of 9.23 $\mathrm{Hz}$ between $\mathrm{H}_{\mathrm{x}}$ and $\mathrm{H}_{\mathrm{y}}{ }^{14}$ Attempts to effect the cycloaddition with C4 methyl (C14) in place were unsuccessful and instead favored a Mukaiyama-Michael adduct likely due to a severe syn-pentane interaction in the cycloaddition transition state. Addition of the methyl group at the expense of additional steps was compensated for by the excellent yield and enantiomeric ratio. The incorrect relative stereochemistry of the $\mathrm{C} 4$ carboxylate proved inconsequential as it was inverted during this methyl addition step.

Wittig olefination of $(-)-\mathbf{1 3}$ proceeded smoothly to access terminal alkene $(+)-\mathbf{1 4}$. Whereas this alkene served as a substrate for a carboxylate-directed Heck reaction ${ }^{11 \mathrm{~b}}$ in our firstand second-generation routes, here it served as a masked, homologated aldehyde (vide infra). Attempts to oxidize silyl enol ether (+)-14 to enone (-)-16 using Saegusa oxidation conditions led to adequate yields, but only at stoichiometric palladium loading ( $40 \%$ yield); attempts to render the oxidation catalytic in palladium were unsuccessful. $\alpha$-Bromination en route (-)-16 led to unexpected silyl enol ether transposition (15, verified by ${ }^{1} \mathrm{H}$ NMR and COSY). ${ }^{15}$ Despite the potential for facile aromatization, $\mathbf{1 5}$ underwent concomitant deprotection and elimination when treated with tetrabutylammonium fluoride to yield (-)-16 in 94\% yield. We did not observe isomerization of the 1,4-diene terminal alkene to the internal position of a conjugated 1,3-diene. Low substrate concentration $(0.03$ $M$ in 15) proved crucial for clean conversion to (-)-16, potentially to disfavor olefin-to-olefin bromonium transfer. ${ }^{16}$
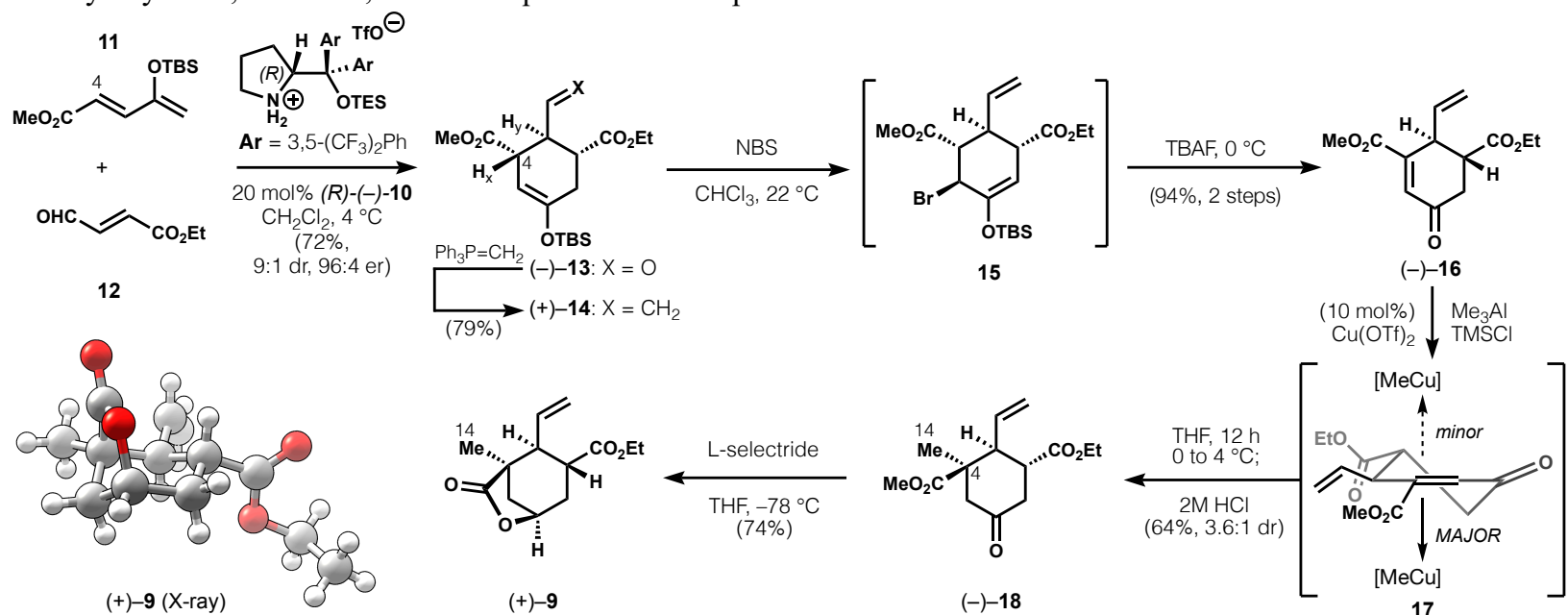

Scheme 1. Short asymmetric synthesis of the collybolide core. 


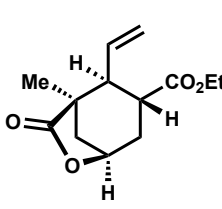

$(+)-9$

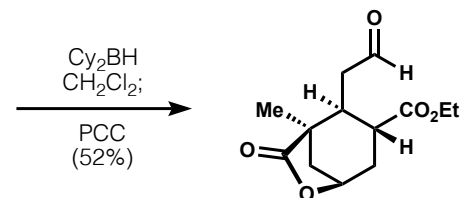

$(+)-19 \quad x^{\ominus}$
$[t-\mathrm{Bu}$

minor: si-facial approach
$t-\mathrm{Bu} 、 \stackrel{\oplus}{\mathrm{N}}-\mathrm{OBz}$

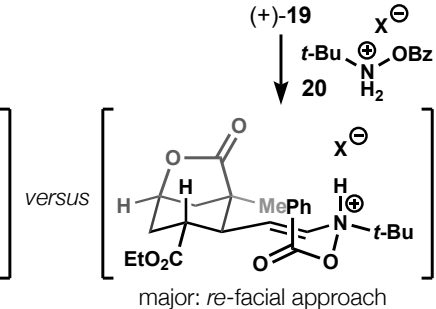

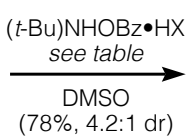

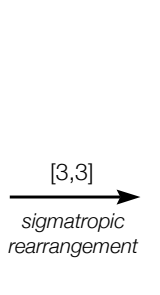

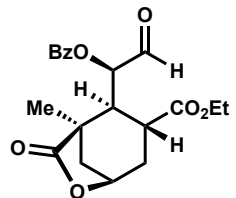
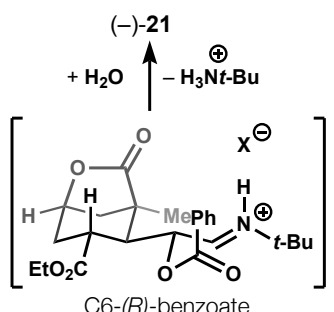

C6-(R)-benzoate

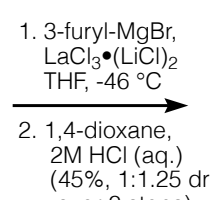

over 2 steps)

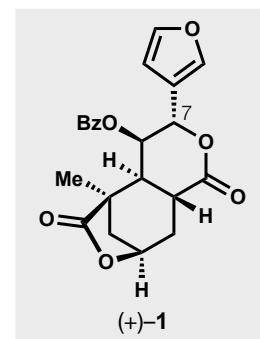

Effect of co-acid on dr

$\begin{array}{lrr}\text { HX } & \text { yield } & \text { dr } \\ \text { TFA } & 77 \% & 2.8: 1 \\ \mathrm{HCl} & 78 \% & 4.2: 1 \\ \text { HOTf } & 25 \% & 2.9: 1\end{array}$

Scheme 2. Completion of collybolide avoids $\mathrm{C} 6-\mathrm{OH}$ intermediate via stereoselective $\mathrm{C}-\mathrm{OBz}$ bond formation.

The challenging $\mathrm{C} 4$ quaternary stereocenter could be accessed via conjugate addition (see Scheme 1), but not using standard approaches (MeLi or MeMgBr in combination with $\mathrm{Cu}(\mathrm{I})$ salts), which either failed to convert (-)-16 or formed 1,2-addition products. To lessen the basicity and increase the Lewis acidity of the methyl pro-nucleophile, we turned to $\mathrm{Me}_{3} \mathrm{Al}^{17}$ in the presence of $(10 \mathrm{~mol} \%) \mathrm{Cu}(\mathrm{I}) \mathrm{Br}^{\circ} \mathrm{SMe}_{2}$, and $\mathrm{TMSCl}$, which furnished the desired conjugate addition product in a modest 1.7:1 dr favoring diastereomer (-)-18 (60\% yield, diastereomers assigned by NOE). The diastereomeric ratio could be improved to $3.6: 1 \mathrm{dr}$ with catalytic $\mathrm{Cu}(\mathrm{II})(\mathrm{OTf})_{2}$ $(10 \mathrm{~mol} \%)^{18}$ in favor of the desired diastereomer $(64 \%$ yield). ${ }^{19}$ Diastereoselectivity may depend on copper aggregation or oxidation states, which could perturb steric interactions with the vinyl substituent en route to $\mathrm{Cu}^{3+}$ insertion products. ${ }^{20}$ Addition of TMSCl prevented further reactivity of the aluminum enolate intermediate; its absence resulted in complex reaction mixtures. Deprotection of the newly formed trimethylsilyl enol ethers occurred upon work-up with $2 \mathrm{M} \mathrm{HCl}$. Diastereoselective reduction of cyclohexanone (-)-18 proceeded smoothly with L-Selectride ${ }^{\circledR}$, resulting in a single diastereomer, which lactonized under the reaction conditions to form $(+)-9$ (75\% yield, relative and absolute stereochemistry assigned by X-ray crystallography, see Scheme 1).

Prosecution of our fourth-generation strategy required oxidation of the terminal alkene to the corresponding aldehyde. Attempts to effect this transformation directly with nitrite-modified aldehyde-selective Wacker oxidation conditions failed to convert starting material, while Wacker oxidation of the alkene under substrate control yielded mixtures of aldehyde and methyl ketone. ${ }^{21}$ Instead, successful oxidation was achieved via a one-pot hydroboration/oxidation approach. Efficient hydroboration of (+)-9 was accomplished exclusively with dicyclohexylborane, whereas $\mathrm{BH}_{3} \bullet \mathrm{SMe}_{2}$ or $\mathrm{Sia}_{2} \mathrm{BH}$ did not hydroborate efficiently and 9-BBN or $\mathrm{BH}_{3} \bullet \mathrm{THF}$ did not allow efficient oxidation. The intermediate trialkylborane was oxidized directly to $(+)-19$ by PCC $(52 \%$ yield $)$. We attribute the difficulties of this alkene oxidation to steric crowding in (+)-9 from both the $\mathrm{C} 4$ methyl and the $\mathrm{C} 9$ ester that flank the terminal olefin. Given the difficulties of the oxidation of vinylfuran 4 (Figure 2a), the steric hindrance of this alkene had proven to be a recurring challenge in this synthesis.

Access to (+)-19 allowed us to skirt this same steric hindrance and probe intra-molecular appendage of the benzoyloxy group (Scheme 2). The intramolecularity of $\alpha$-oxidation would thus solve the problems of instability, inaccessibility and stereoselectivity in a single maneuver. To carry out the benzoyloxylation, we turned to ammonium ion 20, explored extensively by the Tomkinson group in the chemoselective $\alpha$ oxygenations of aldehydes and ketones. ${ }^{22}$ The mechanism proposed for this transformation involves condensation to the iminium, tautomerization to the enamine and [3,3]-sigmatropic rearrangement to give the $\alpha$-benzoyloxy imine, which hydrolyzes in situ or upon workup. The development of this reagent hinges on literature precedent from House and Cummins on the spontaneous rearrangement of acylated oxime or nitrone intermediates, respectively. ${ }^{23} \mathrm{~A}$ multi-hetero Claisen rearrangement proceeding through a chair-like transition state (i.e. $(+)-19$ to (-)-21) suggests two competing transition state conformers leading to the major and minor diastereomers. ${ }^{24}$ Diastereoselectivity was improved by variation of acid coreagents, with $\mathrm{HCl}$ leading to the highest dr (4.2:1) (see Table in Scheme 2). This dependence of $\mathrm{dr}$ on acid suggests that the acid either affects the equilibrium ratio of enamine conformations or changes the relative rates of rearrangement. ${ }^{25}$ Although [3,3]-rearrangement is a precedented mechanism, we cannot rule out an alternative bimolecular benzoate transfer. Nevertheless, only furan addition and lactonization now separated (-)-21 from $\mathbf{1}$.

3-Metallofuran addition into an aldehyde had proven to be an effective approach to the related aryl- $\delta$-lactone motif of salvinorin A. ${ }^{26}$ Organotitanium reagents, in particular, exhibited high chemoselectivity and, in the presence of scalemic titanium Lewis acids, diastereoselectivity. ${ }^{27}$ In our hands, 3furyltitanium reagents failed to deliver $\mathbf{1}$, however, resulting instead in titanium alkoxide addition to the aldehyde. Furyllithium and magnesium halides were unsuccessful, either resulting in recovered starting material or complex reaction mixtures. Organolanthanides, however, proved effective, likely due to the high nucleophilicity and oxophilicity but low basicity relative to organolithium and -magnesium counterparts. We first explored the organocerium, but operational difficulties of the hygroscopic and poorly soluble cerium(III) chloride salt led to irreproducible results between batches of reagent. The organolanthanum, however, could be formed by transmetalation of the corresponding Grignard reagent using THF solutions of $\mathrm{LaCl}_{3} \cdot 2 \mathrm{LiCl}$ and reproducibly yielded collybolide (+)1 and 7-epi-collybolide (7-epi-1) (1:1.25) ${ }^{28}$ Attempts to vary diastereoselectivity by increasing the temperature were unsuccessful. Fortunately, Potier and coworkers found that trifluoroacetic acid established an equilibrium between collybolide and 7-epi-collybolide $\left(1: 1\right.$ at $\left.72{ }^{\circ} \mathrm{C}\right)$, which allows material to be cycled toward collybolide. ${ }^{1} \mathrm{H}$ and ${ }^{13} \mathrm{C}$ NMR spectra of synthetic materials were identical 
a. ${ }^{1} \mathrm{H}$ NMR spectra comparison

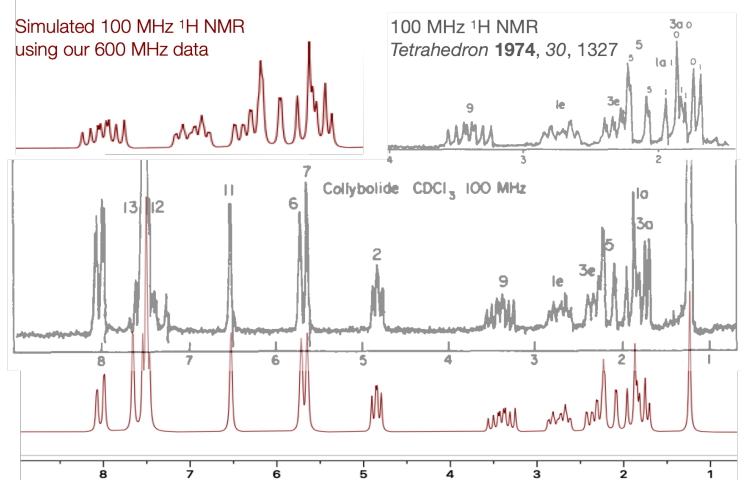

c. Assessment of agonist activity at hKOR.
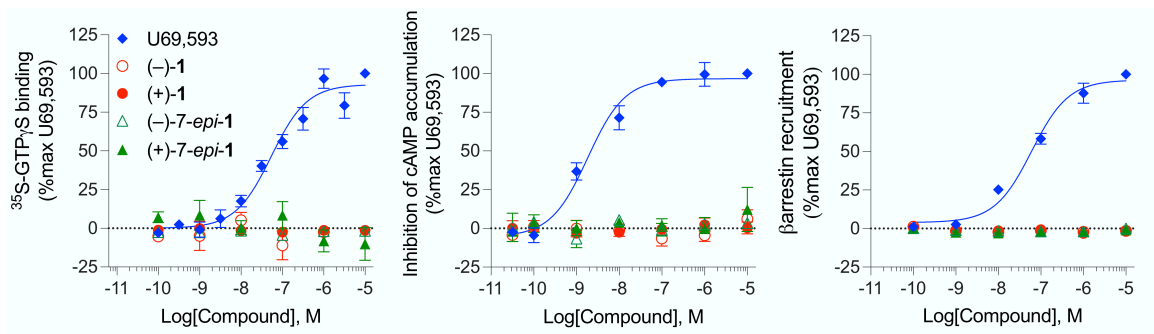

d. Assessment of antagonist activity at hKOR.

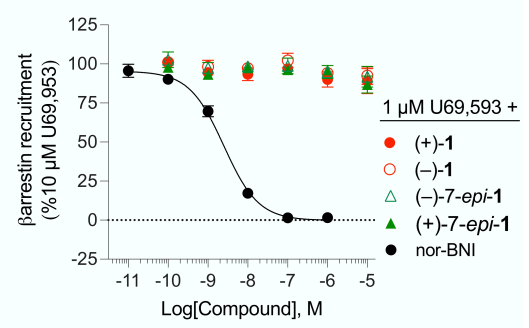

Figure 3. Neither enantiomer of collybolide (1) or 7-epi-1 activate or inhibit kappa-opioid receptor signaling. a. The ${ }^{1} \mathrm{H}$ NMR data report of synth-(+)-1 matches nat-(+)-1 and its $600 \mathrm{MHz}$ data can be simulated at $100 \mathrm{MHz}$ to produce aligned spectra for visual comparison. b. The rapidity of complexity increase, depicted on a Gantt chart, allowed the synthesis of $(-)-1$ in 12 days. c. Stimulation of hKOR using $(+)-1,(-)-1,(-)-7-e p i-1$ and $(+)-7-e p i-1\left(10^{-10}-10^{-5} \mathrm{M}\right)$ relative to the control agonist U69,593 in (left) a $\left[{ }^{35} \mathrm{~S}\right] \mathrm{GTP} \gamma \mathrm{S}$ binding assay performed in hKOR-CHO cell membranes; (center) inhibition of forskolin-stimulated cAMP accumulation in CHO-hKOR cells; (right) $\beta$ arrestin2 recruitment assay (U20S hKOR DiscoveRx PathHunter ${ }^{\circledR}$ ). d. Antagonism of $1 \mu \mathrm{M}$ U69,593 stimulated $\beta$-arrestin2 recruitment with the KOR antagonist, norBNI; collybolide does not antagonize the response. Experimental details can be found in Ref. 29.

in all aspects to those of naturally-occurring $\mathbf{1}$ and 7-epi-1 (see SI and Figure 3a). Optical rotation and circular dichroism spectroscopy correlated the absolute configuration imparted by (R)-(-)-10 to synthetic (+)-1 with the data reported for naturally-occurring collybolide, showing the materials were identical in all respects, with one major caveat.

Access to (+)-1 allowed its independent analysis in human kappa-opioid receptor (hKOR) $\left[{ }^{35} \mathrm{~S}\right] \mathrm{GTP} \gamma \mathrm{S}$ binding assays (see Figure 3c). To our surprise, $(+)-\mathbf{1}$ demonstrated no increase in $\left[{ }^{35} \mathrm{~S}\right] \mathrm{GTP} \gamma \mathrm{S}$ binding over basal activity, in contrast to the 2016 report of low nanomolar affinity and potency. ${ }^{2}$ To further investigate the pharmacological properties of $(+)-\mathbf{1}$, we also tested whether agonism could be revealed in other assays; however, there was no evidence that $(+)-\mathbf{1}$ activated hKOR in assays measuring inhibition of adenylyl cyclase or the recruitment of $\beta$-arrestin2. Moreover, to assure that we were not missing low efficacy partial agonism, we tested whether (+)-1 could antagonize $\beta$-arrestin 2 recruitment induced by U69,593; however, again no efficacy for this system was observed. The lack of efficacy associated with synthetic (+)-1 might have been caused by synthesis of the incorrect unnatural enantiomer. Although the optical rotation and CD spectra of synth-1 agreed with isolated nat-1, values in either sample might be overridden by an impurity with large circular birefringence of the opposite rotation as 1. Existing X-ray crystal structures of 1 and 9-epi-1 reported inconclusive Flack parameters ${ }^{8}$ that prevent confidence in the assignment. ${ }^{9}$ In contrast, the structure parameters of (+)-9 (X-ray) and others (see SI) allowed conclusive assignment of absolute stereochemistry in the series from $(R)-(-)-\mathbf{1 0}$.
Given the brevity of our synthesis and the unexpected inactivity of synth-(+)-1, we decided to synthesize the opposite enantiomer, (-)-1. Normally a major undertaking, the entire synthesis from $(S)-\mathbf{1 0} \cdot$ HOTf was performed in only 12 days. The Gantt chart (Figure 3b) depicts the ease with which the route attains the 443 molecular bits (mcbits) of 1 from low complexity building blocks 11 and 12 (95 and 171 mcbits, respectively). Access to ent-(-)-1, the enantiomer of the absolute configuration assigned to nat-1, allowed parallel assays of (+)-1 and (-)-1 to identify which antipode might be responsible for KOR agonism. To our dismay, neither enantiomer of $\mathbf{1}$ or 7-epi-1 indicated KOR agonism as evident through $\left[{ }^{35} \mathrm{~S}\right] \mathrm{GTP} \gamma \mathrm{S}$ functional assay or inhibition of cAMP accumulation. These data significantly affect ongoing research and expectations from multiple laboratories involved in opioid research related to the collybolides.., 30

Conclusion Whereas biological activity has been wrongly ascribed to natural products when assay readouts are uncertain or impurities are present, ${ }^{31}$ the conflict described here represents an unusual case in which multiple, well-established biochemical assays are incorrectly assigned. The explanation we favor for this mischaracterization is error in the assays themselves $;^{32}$ the original dose-response data ${ }^{2}$ did not fit a standard sigmoidal curve, both for $\mathbf{1}$ and the positive control, salvinorin A. Other explanations might include the presence of an unidentified impurity in isolates (ergosterol partly co-eluted with collybolide), ${ }^{\text {la }}$ degradation of collybolide during storage to an active congener that was not removed by purification, or degradation of collybolide to an active congener during sample preparation or under the assay conditions. Related to these two latter possibilities is the recent discovery that derivatives of columbin, another furano- $\delta$-lactone terpenoid, agonize KOR 
even though columbin itself does not. ${ }^{33}$ If degradation products of collybolide are KOR agonists, our ability to quickly reach collybolide chemical space (Figure $3 b$ ) positions us well to access, modify and improve these congeners. Furthermore, the observed in vivo activity of collybolide in mouse studies raises the possibility that $\mathbf{1}$ may be a potent antipruritic, but its target has been incorrectly assigned. ${ }^{34}$ For the time being, excitement over identification of a new non-nitrogenous, KORselective agonist with the same clinical potential as SalA seems to have been misplaced. The independent syntheses and assays reported here have excluded collybolide as a KOR agonist and have begun a search for the truth.

\section{ASSOCIATED CONTENT}

\section{Supporting Information}

The Supporting Information is available free of charge on the ACS Publications website. Experimental procedures and spectra are available (pdf).

\section{AUTHOR INFORMATION}

\section{Corresponding Author}

*rshenvi@scripps.edu

\section{Present Addresses}

†Chemistry Department, Massachusetts Institute of Technology, 77 Massachusetts Ave., Cambridge, MA 02139

\section{ACKNOWLEDGMENT}

Arnold Rheingold, Milan Gembicky, Jack Bailey and Erika Samolova are acknowledged for X-ray crystallographic analysis. We thank Dr. Marcus Jaegar and the Kelly lab for use of their circular spectropolarimeter. We thank Dr. L. Pasternack and Dr. D.-H. Huang for NMR assistance. We gratefully acknowledge Dr. Thomas Munro for insightful comments regarding a possible connection between antipruritic activity and $\mathrm{Ca}^{2+}$ channel inhibitors. The Scripps Automated Synthesis Facility at Scripps performed separations and analysis of key intermediates. Support was provided by the National Institutes of Health (R35 GM122606, TL1TR002551, DA031927 and DA048490) and the Skaggs Graduate School (fellowship to S.M.F.).

\section{REFERENCES}

1. a) Bui, A.-M.; Cavé, A.; Janot, M.-M.; Parello, J.; Potier, P. Isolement et Analyse Structurale du Collybolide, Nouveau Sesquiterpene Extrait de Collybia Maculata Alb. et sch. ex fries (Basidiomycetes). Tetrahedron 1974, 30, 1327-1336; b) Castronovo, F.; Clericuzio, M.; Toma, L.; Vidari, G. Fungal metabolites. Part 45: The sesquiterpenes of Collybia maculata and Collybia peronata. Tetrahedron 2001, 2791-2798.

2. Gupta, A.; Gomes, I.; Bobeck, E. N.; Fakira, A. K.; Massaro, N. P.; Sharma, I.; Cave, A.; Hamm, H. E.; Parello, J.; Devi, L. A. Collybolide is a novel biased agonist of $\kappa$-opioid receptors with potent antipruritic activity. Proc. Natl. Acad. Sci., USA 2016, 113, 6041-6046.

3. Stahl, E. L.; Bohn, L. M. Low Intrinsic Efficacy Alone Cannot Explain the Improved Side Effect Profiles of New Opioid Agonists. Biochemistry

2021,
4. Nakao, K.; Mochizuki, H. Nalfurafine hydrochloride: A new drug for the treatment of uremic pruritus in hemodialysis patients. Drugs Today 2009, 45, 323.

5. a) Badal, S.; Turfus, S.; Rajnarayanan, R.; Wilson-Clarke, C.; Sandiford, S. L. Analysis of natural product regulation of opioid receptors in the treatment of human disease. Pharmacol. Ther. 2018, 184, 51-80; b) Chakraborty, S.; Majumdar, S. Natural products for the treatment of pain: Chemistry and pharmacology of salvinorin A, mitragynine, and collybolide. Biochemistry 2021, 60, 1381-1400; c) Faouzi, A.; Varga, B. R.; Majumdar, S. Biased Opioid Ligands. Molecules 2020, 25, 4257-4291; d) Ji, M.-J.; Yang, J.; Gao, Z.-Q.; Zhang, L.; Liu, C. The Role of the Kappa Opioid System in Comorbid Pain and Psychiatric Disorders: Function and Implications. Front. Neuroscience 2021, 15, https://doi.org/10.3389/fnins.2021.642493; e) Kenakin, T. Biased receptor signaling in drug discovery. Pharmacol. Rev. 2019, 71, 267-315; f) Mores, K. L.; Cummins, B. R.; Cassell, R. J.; van Rijn, R. M. A Review of the Therapeutic Potential of Recently Developed G Protein-Biased Kappa Agonists. Front. Pharmacol. 2019, https://doi.org/10.3389/fphar.2019.00407; g) Paton, K. F.; Atigari, D. V.; Kaska, S.; Prisinzano, T.; Kivell, B. M. Strategies for Developing kappa Opioid Receptor Agonists for the Treatment of Pain with Fewer Side Effects. J. Pharmacol. Exp. Ther. 2020, $375,332-348$.

6. Taylor, G. T.; Manzella, F. Kappa Opioids, Salvinorin A and Major Depressive Disorder. Curr. Neuropharmacol. 2016, 14, 165-176.

7. Bohn, L. M.; Aube, J. Seeking (and Finding) Biased Ligands of the Kappa Opioid Receptor. ACS Med. Chem. Lett. 2017, 8, 694700 .

8. Flack, H. D.; Bernardinelli, G. The use of X-ray crystallography to determine absolute configuration. Chirality 2008, 20, 681-690.

9. See CCDC 1425246 and CCDC 1425247.

10. Baker, M.; Demoret, R.; Ohtawa, M.; Shenvi, R. A. Concise Asymmetric Synthesis of (-)-Bilobalide. Nature 2019, 575, 643646.

11. a) Roach, J. J.; Sasano, Y.; Schmid, C. L.; Zaidi, S.; Katritch, V.; Stevens, R. C.; Bohn, L. M.; Shenvi, R. A. Dynamic Strategic Bond Analysis Yields a Ten-Step Synthesis of 20-nor-Salvinorin A, a Potent $\kappa$-OR Agonist. ACS Cent. Sci. 2017, 3, 1329-1336; b) Huffman, T. R.; Wu, Y.; Emmerich, A.; Shenvi, R. A. Intermolecular Heck Coupling with Hindered Alkenes Directed by Potassium Carboxylates. Angew. Chem. Int. Ed. 2019, 58, 2371-2376.

12. a) Marigo, M.; Wabnitz, T. C.; Fielenbach, D.; Jorgensen, K. A. Enantioselective organocatalyzed $\alpha$ sulfenylation of aldehydes. Angew. Chem. Int. Ed. 2005, 44, 794-797; b) Hayashi, Y.; Gotoh, H.; Hayashi, T.; Shoji, M. Diphenylprolinol Silyl Ethers as Efficient Organocatalysts for the Asymmetric Michael Reaction of Aldehydes and Nitroalkenes. Angew. Chem. Int. Ed. 2005, 44, 4212-4215.

13. You, L.; Liang, X. T.; Xu, L. M.; Wang, Y. F.; Zhang, J. J.; Su, Q.; Li, Y. H.; Zhang, B.; Yang, S. L.; Chen, J. H.; Yang, Z. Asymmetric Total Synthesis of Propindilactone G. J. Am. Chem. Soc. 2015, 137, 10120-10123.

14. a) Gotoh, H.; Hayashi, Y. Diarylprolinol Silyl Ether as Catalyst of an exo-Selective, Enantioselective Diels-Alder Reaction. Org. Lett. 2007, 9, 2859-2862; b) Cassaidy, K. J.; Rawal, V. H. Enantioselective Total synthesis of (+)-Heilonine. $J$. Am. Chem. Soc. 2021, 143, 16394-16400; c) Kano, T.; Tanaka, Y.; Maruoka, K. exo-Selective Asymmetric Diels-Alder Reaction Catalyzed by Diamine Salts as Organocatalysts. Org. Lett. 2006, $8,2687-2689$. 
15. a) Tsuchimochi, I.; Kitamura, Y.; Aoyama, H.; Akai, S.; Nakai, K.; Yoshimitsu, T. Total synthesis of (-)-agelastatin A: an $\mathrm{S}_{\mathrm{H}} 2$ ' radical azidation strategy. Chem. Commun. 2018, 54, 98939896; b) Pu, L. Y.; Yang, F.; Chen, J. Q.; Xiong, Y.; Bin, H. Y.; Xie, J. H.; Zhou, Q. L. Enantioselective Total Syntheses of Pentacyclic Homoproaporphine Alkaloids. Org. Lett. 2020, 22, 7526-7530; c) Hambly, G. F.; Chan, T. H. Reactions of Enol Silyl Ethers with N-Halosuccinomide - A Stepwise Process. Tet. Lett. 1986, 27, 2563-2566.

16. Denmark, S. E.; Burk, M. T.; Hoover, A. J. On the Absolute Configurational Stability of Bromonium and Chloronium Ions. $J$. Am. Chem. Soc. 2010, 132, 1232-1233.

17. a) Westermann, J.; Nickisch, K. Copper-Catalyzed 1,4Additions of Trialkylaluminum Compounds to Enones. Angew. Chem. Int. Ed. 1993, 32, 1368-1370; b) Brown, M. K.; May, T. L.; Baxter, C. A.; Hoveyda, A. H. All-Carbon Quaternary Stereogenic Centers by Enantioselective $\mathrm{Cu}$-Catalyzed Conjugate Additions Promoted by a Chiral N-Heterocyclic Carbene. Angew. Chem. Int. Ed. 2007, 46, 1097-1100; c) May, T. L.; Brown, M. K.; Hoveyda, A. H. Enantioselective synthesis of all-carbon quaternary stereogenic centers by catalytic asymmetric conjugate additions of alkyl and aryl aluminum reagents to five-, six-, and seven-membered-ring beta-substituted cyclic enones. Angew. Chem. Int. Ed. 2008, 47, 7358-7362; d) d'Augustin, M.; Palais, L.; Alexakis, A. Enantioselective copper-catalyzed conjugate addition to trisubstituted cyclohexenones: construction of stereogenic quaternary centers. Angew. Chem. Int. Ed. 2005, 44, 1376-1378.

18. Sakata, H.; Aoki, Y.; Kuwajima, I. A Remarkable Catalytic Effect of $\mathrm{Cu}(\mathrm{II})$ Species for Conjugate Addition of Grignard Reagents to $\alpha, \beta$-Unsaturated Esters. Tet. Lett. 1990, 31, 11611164 .

19. de Vries, A. H. M.; Meetsma, A.; Feringa, B. L. Enantioselective Conjugate Addition of Dialkylzinc Reagents to Cyclic and Acyclic Enones Catalyzed by Chiral Copper Complexes of New Phosphorus Amidites. Angew. Chem. Int. Ed. 1996, 35, 2374-2376.

20. Bertz, S. H.; Hardin, R. A.; Murphy, M. D.; Ogle, C. A.; Richter, J. D.; Thomas, A. A. Rapid injection NMR reveals $\eta^{3}$ ' $\pi$ allyl' $\mathrm{Cu}(\mathrm{III})$ intermediates in addition reactions of organocuprate reagents. J. Am. Chem. Soc. 2012, 134, 9557-9560.

21. a) Kim, K. E.; Li, J.; Grubbs, R. H.; Stoltz, B. M. Catalytic Anti-Markovnikov Transformations of Hindered Terminal Alkenes Enabled by Aldehyde-Selective Wacker-Type Oxidation. J. Am. Chem. Soc. 2016, 138, 13179-13182; b) Ning, X. S.; Wang, M. M.; Yao, C. Z.; Chen, X. M.; Kang, Y. B. tert-Butyl Nitrite: Organic Redox Cocatalyst for Aerobic Aldehyde-Selective Wacker-Tsuji Oxidation. Org Lett 2016, 18, 2700-2703.

22. a) Beshara, C. S.; Hall, A.; Jenkins, R. L.; Jones, T. C.; Parry, R. T.; Thomas, S. P.; Tomkinson, N. C. A simple method for the $\alpha$-oxygenation of aldehydes. Chem. Commun. 2005, 14781480; b) Smithen, D. A.; Mathews, C. J.; Tomkinson, N. C. Asymmetric $\alpha$-oxyacylation of cyclic ketones. Org. Biomol. Chem. 2012, 10, 3756-3762; c) Beshara, C. S.; Hall, A.; Jenkins, R. L.; Jones, K. L.; Jones, T. C.; Killeen, N. M.; Taylor, P. H.; Thomas, S. P.; Tomkinson, N. C. A General Method for the $\alpha-$ Acyloxylation of Carbonyl Compounds. Org. Lett. 2005, 7, 57295732; For examples of $\mathbf{2 0}$ in total synthesis, see d) Sherwood, T. C.; Trotta, A. H.; Snyder, S. A. A Strategy for Complex Dimer Formation when Biomimicry Fails: Total Synthesis of Ten Coccinellid Alkaloids. J. Am. Chem. Soc. 2014, 136, 9743-9753; e) Nugent, J.; Banwell, M. G.; Schwartz, B. D. Total Synthesis of the Illicium-Derived Sesquineolignan Simonsol C. Org. Lett. 2016, 18, 3798-3801.
23. a) House, H. O.; Forrest, A. R. Use of ketoxime derivatives to prepare $\alpha$-acetoxy ketones. J. Org. Chem. 1969, 34, 1430-1439; b) Cummins, C. H.; Coates, R. M. $\alpha$-Oxygenation of aldehydes and cyclic ketones by acylation-rearrangement of nitrones. J. Org. Chem. 1983, 48, 2070-2076.

24. Tabolin, A. A.; Ioffe, S. L. Rearrangement of $N$-oxyenamines and related reactions. Chem. Rev. 2014, 114, 5426-5476.

25. Mannschrek, A.; Koelle, U. Internal Rotation about the $=\mathrm{C}-\mathrm{N}$ Bond in Enamines and the $=\mathrm{N}-\mathrm{N}$ Bond in Hydrazones. Tet. Lett. 1967, 863-867.

26. Hill, S. J.; Brion, A. U. C. M.; Shenvi, R. A. Chemical syntheses of the salvinorin chemotype of KOR agonist. Nat. Prod. Rep. 2020, 37, 1478-1496.

27. a) Line, N. J.; Burns, A. C.; Butler, S. C.; Casbohm, J.; Forsyth, C. J. Total Synthesis of (-)-Salvinorin A. Chem. Eur. J. 2016, 22, 17983-17986; b) Sibi, M. P.; He, L. Application of Enantioselective Radical Reactions: Synthesis of (+)Ricciocarpins A and B. Org. Lett. 2004, 6, 1749-1752.

28. Krasovskiy, A.; Kopp, F.; Knochel, P. Soluble Lanthanide Salts $\left(\mathrm{LnCl}_{3} \cdot 2 \mathrm{LiCl}\right)$ for the Improved Addition of Organomagnesium Reagents to Carbonyl Compounds. Angew. Chem. Int. Ed. 2006, 45, 497-500.

29. Ho, J.-H.; Stahl, E. L.; Schmid, C. L.; Scarry, S. M.; Aube, J.; Bohn, L. M. G protein signaling-biased agonism at the $\kappa-$ opioid receptor is maintained in striatal neurons. Sci. Signal. 2018, $11,10.1126 /$ scisignal.aar4309.

30. a) Southgate, E. H. Dearomative Transformations in Synthesis: I. Dearomative Dihydroxylation with Arenophiles II. Total Synthesis of Lycoricidine and Narciclasine III. Towards the Total Synthesis of Collybolide. Ph.D., University of Illinois at Urbana-Champaign, Urbana, Illinois, 2019; b) Sharma, I. In Structure-Activity Relationships on Collybolide: Discovery of a Potent Kappa-Opioid Agonist with Enhanced Metabolic Stability, KappaCon 2021, April 7-9, 2021; 2021; c) Sharma, I., Design and Synthesis of Collybolide Probes for Kappa-Opioid Receptor. National Institutes of Health (NIH): Norman, OK, 2019.

31. Pauli, G. F.; Chen, S. N.; Friesen, J. B.; McAlpine, J. B.; Jaki, B. U. Analysis and purification of bioactive natural products: the AnaPurNa study. J. Nat. Prod. 2012, 75, 1243-1255.

32. We have corresponded with the authors of Ref. 2, who graciously sent their assay protocols and provided additional insight. Their sample of collybolide was described as sticky and led them to treat all glass and plastic with surface modifying agents. We did not observe insolubility of 1 in DMSO or in serial dilutions. The ClogP (ChemOffice) is calculated to be 1.99, which correlates well with its extrapolated $\log \mathrm{K}_{\mathrm{w}}$ of 3.8. See Supporting Information.

33. Yilmaz, A.; Crowley, R. S.; Sherwood, A. M.; Prisinzano, T. E. Semisynthesis and Kappa-Opioid Receptor Activity of Derivatives of Columbin, a Furanolactone Diterpene. J Nat Prod 2017, 80, 2094-2100.

34. Collybolide has been identified as an L-type calcium channel inhibitor in isolated rat aorta: Hansske, F.; Paululat, T.; Gerlitz, M.; Grün-Wollny, I. Medicaments containing collybolides. U.S. Patent WO2005051376, June 9, 2005

\section{Graphical Abstract}




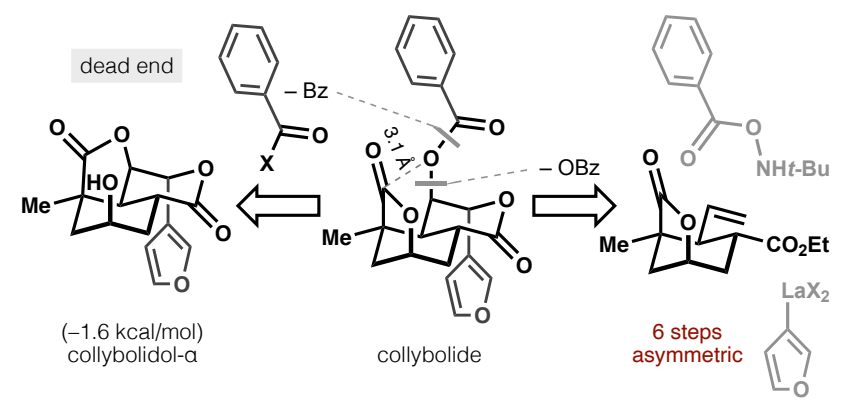

Synthesis of both enantiomers and biological assay 